\title{
Binary interactions on the RGB: Dusty post-RGB stars
}

\author{
Devika Kamath $^{1}$, Hans Van Winckel ${ }^{1}$ and Peter Wood ${ }^{2}$ \\ ${ }^{1}$ Instituut voor Sterrenkunde, K.U.Leuven, Celestijnenlaan 200D bus 2401, \\ B-3001 Leuven, Belgium \\ email: devika@ster.kuleuven.be \\ ${ }^{2}$ Research School of Astronomy and Astrophysics, Mount Stromlo Observatory, Weston Creek, \\ ACT 2611, Australia \\ email: wood@mso.anu.edu.au
}

\begin{abstract}
It is widely accepted that binary interactions are responsible for the shaping of planetary nebula. However, these binary interactions and evolutionary channels are poorly understood. Our recent study revealed a newly discovered population of low-luminosity, lowmetallicity, likely binaries in the Magellanic Clouds: dusty post-RGB stars. They are likely to have evolved off the RGB via binary interaction. In this paper we present preliminary results of the first radial velocity monitoring of the post-RGB stars in the Small Magellanic Cloud (SMC) and the implications on stellar (binary) evolution. We also investigate their link, if any, to the planetary nebulae systems.
\end{abstract}

Keywords. techniques: radial velocities, stars: AGB and post-AGB, (stars:) binaries: general, stars: evolution, stars: Population II, (ISM:) planetary nebulae: general, Galaxy: general, (galaxies:) Magellanic Clouds.

\section{Introduction}

Dusty post-red giant branch (post-RGB) stars are a new class of evolved objects that lie below the tip of the RGB. These are dusty (with large infrared excesses $\geqslant 8 \mu \mathrm{m}$ ) low metallcity, evolved objects with luminosities below the tip of the RGB with an $M_{\text {bol }}($ TRGB $)=-3.6$ based on studies by Frogel et al. (1983). These objects are very similar to the well known post-AGB stars, see Van Winckel (2003), in terms of their stellar parameters $\left(T_{\text {eff }}, \log g,[\mathrm{Fe} / \mathrm{H}]\right.$ and $\left.\mathrm{E}[B-V]\right)$ and circumstellar environment characteristics. However, they have luminosities less than the RGB tip $\left(\sim 2500 \mathrm{~L}_{\odot}\right)$. It is therefore likely that these objects are evolved, dusty post-RGB stars. These objects were first discovered in the Magellanic Clouds (MCs) by Kamath et al. (2016) during the search for post-AGB stars in the MCs (Kamath et al. 2014; Kamath et al. 2015).

During the RGB phase (where luminosities are $<2500 \mathrm{~L}_{\odot}$ ), single-star mass loss is insufficient to remove the H-rich envelope and produce a dusty post-RGB star (Vassiliadis \& Wood 1993). The only way large amounts of mass loss and premature evolution off the RGB can occur is via binary interaction (Han et al. 1995). In the Galaxy, the binaries among the post-AGB stars all have a common property: their SEDs display a clear nearinfrared excess indicating that circumstellar dust must be close to the central star, near sublimation temperature (Van Winckel 2003). It is now well established that this feature in the SED indicates the presence of a stable compact circumbinary disc (De Ruyter et al. 2006; Bujjarrabal et al. 2013; Hillen et al. 2013). These Galactic post-AGB binaries show orbital periods between 50 and 2000 days. It is possible that some of the Galactic post-AGB stars might be post-RGB stars but since we do not have accurate distances 

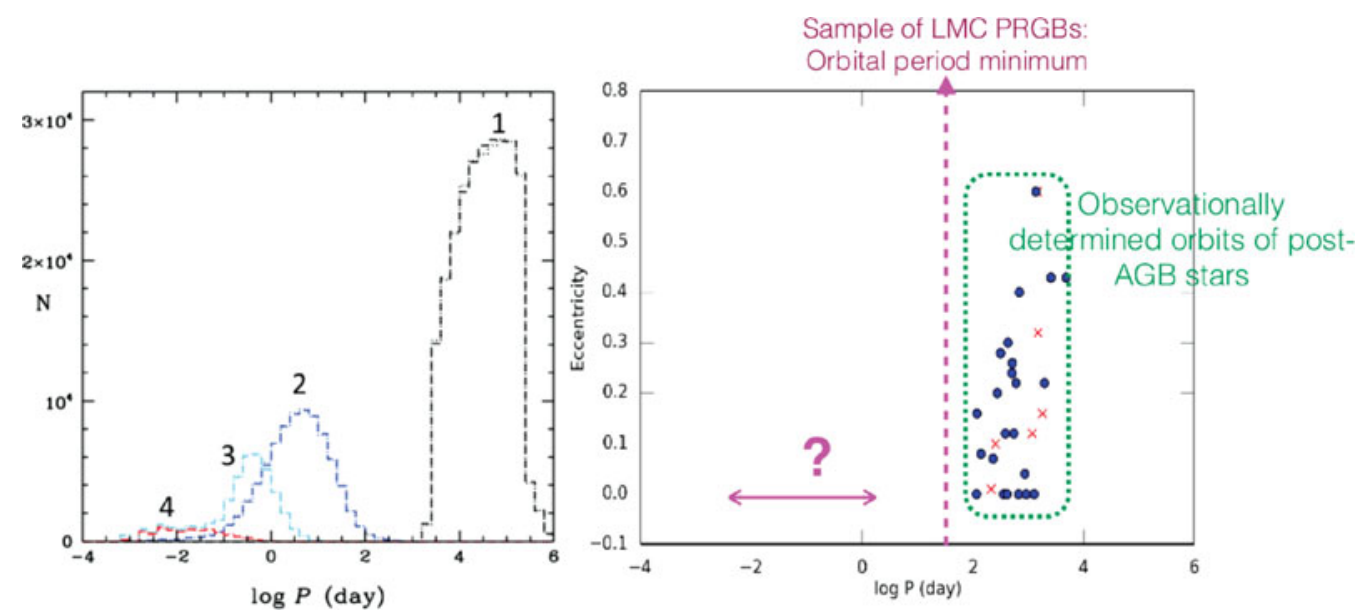

Figure 1. Left: The results of the population synthesis models which were normalised to the sequence E ellipsoidal variables of the Large Magellanic Cloud (LMC). The distribution of intermediate period binary post-AGB systems is given in black (curve1), the close binary post-AGB stars in blue (curve 2), the post-RGB and post-EAGB binaries in cyan/grey (curve 3) and the double degenerate secondaries in red (curve 4). Figure from Nie et al. (2012). Right: Our observed orbits for the Galactic systems (Van Winckel et al. 2009) and the SMC post-RGB stars. The red crosses indicate systems where the companion is certainly too massive to be a WD. The blue-filled circles represent systems where the companion can either be non-evolved or a cold WD. All orbits fall in the period range not predicted by the models. We note that some of these post-AGB stars could very well be binaries but the lack of accurate distances to these objects does not allow us to identify the possible post-RGBs amongst the post-AGB sample. The pink-dashed vertical line marks the minimum observed orbital period for the post-RGB stars in the SMC. As shown this is in stark contrast to that predicted by the models (denoted by the pink horizontal dashed double-headed arrow).

to the Galactic objects, identifying post-RGB systems in the Galaxy is not possible for now.

However, from the post-RGB stars in the MCs, we find that many of our new post-RGB candidates show similar disc-type SEDs to the post-AGB stars, pointing to binarity in these objects. Furthermore, in our recent study (Kamath et al. 2016) we were able to show that the likely precursors of these dusty post-RGB stars are the sequence-E variables, which are close binary red giants that show ellipsoidal light variations (Nicholls et al. 2012). In this paper, we present the preliminary results from the first radial velocity monitoring of the SMC post-RGB stars, their implications for binary evolution and we also aim to investigate the evolutionary link between these objects and planetary nebulae $(\mathrm{PNe})$.

\section{Radial Velocity Monitoring of post-RGB stars in the SMC}

We recently initiated a radial velocity monitoring study of post-RGB stars in the SMC, to confirm their new channel of post-RGB (likely) binary evolution. We used X-shooter on UT2 at ESO, to obtain high-quality spectra $(\mathrm{R}=10000$ and a $\mathrm{S} / \mathrm{N}$ per resolution element of $\sim 70-80$ ) of a carefully selected subset of our newly identified SMC post-RGB candidates, which covered the entire RGB luminosity space as well as showed diverse SED characteristics. We received 2 epochs of data, covering a period of 60 days, (ESO Proposal ID: 097.D-0800(A)). 
On analysing the data, we were unable to find any radial velocity changes. This shows that these post-RGB objects, if they are binaries, are likely to have orbital periods greater than 60 days. This makes them, once again, similar to the post-AGB systems with intermediate to wide orbits (Van Winckel et al. 2007).

However, population synthesis models (Nie et al. 2012) normalised to the LMC binary red-giants (the likely progeny of these dusty post-RGB objects), predict that majority of our systems are likely to have orbital periods in the range of $\sim 25$ to 100 days, or up-to 500 days for the higher luminosity objects. We are continuing our radial velocity monitoring of these objects to cover the entire predicted orbital period range of 500 days. However, our preliminary results shows that these post-RGB systems are unlikely to evolve as the close-binaries or spiral-in systems, as predicted. In Fig 1, we show the discrepancy between the observed periods of the Galactic post-AGB and the SMC postRGB binaries and the predictions from population synthesis models.

\section{Do post-RGB stars evolve into planetary nebulae?}

To establish a link between our newly identified post-RGB systems and the well known planetary nebula, we perform a simple exercise.

The lifetime of the post-RGB phase is determine by the rate of consumption of the $\mathrm{H}$-envelope as well as the dissipation of the circumstellar environment (most likely a circumbinary disc). The observational lifetime of the post-RGB phase is $\sim 1.26 \times 10^{5}$ years. This agrees rather well, within the errors, with the predicted lifetime of $\sim 1.01 \times 10^{5}$ years (based on a stellar evolutionary model without mass-loss at $\log L / \mathrm{L}_{\odot}=3.0$ ). The observed lifetime indicates a small amount of mass-accretion is required on the RGB. Post-RGB systems are expected to have discs which sustains the re-accretion. The discs around these stars may provide a reservoir of hydrogen that can be accreted back on to the high-luminosity star to fuel nuclear burning for an extended period, at the same time keeping the hydrogen envelope thick enough to maintain a relatively low Teff, which is insufficient to excite a PN. The lifetime of the disc (in the case of post-AGB systems but we expect this for post-RGB systems as well) is $\sim 10^{4}$ years (Bujarrabal et al. 2015) for a central stellar mass of $1.1 \mathrm{M}_{\odot}$ and envelope mass of $4 \times 10^{-3} \mathrm{M}_{\odot}$. Therefore, taking the two lifetimes together, towards the end of the PRGB phase, when $T_{\text {eff }}$ is high enough, there is probably no material left to ionise. This could imply that a common envelope interaction leading to some sort of stable mass transfer, as expected from post-RGB binaries may actually not produce a PNe. We note that the estimated and predicted birthrates are subject to several uncertainties.

\section{Conclusions}

A likely binary interaction on the RGB terminates the RGB evolution for some red giant binaries producing dusty post-RGB stars. Observationally, based on a very small sample, these systems have orbital periods greater than 60 days. This however is in contrast to the predicted orbital periods for these objects. This shows that we need to find out why our objects do not spiral-in or produce close binary systems. Furthermore, we need to identify what determines whether a system evolves into a spiral-in or a close binary or via stable mass transfer. To answer these questions we intend to carry out a systematic study of post-AGB and post-RGB binaries and compare their estimated orbital parameters to those predicted by binary evolution models. Our studies also show that a disc is an important ingredient to explain our system. However, it is still unclear 
as to how these discs evolve and the impact they have on the evolution of the postAGB/post-RGB star. Based on the lifetime of post-RGB stars and the estimated lifetime of the circumbinary disk, we find that it is unlikely that these post-RGB stars would evolve into PNe. Likely progeny could be a white dwarf with a helium core, a cataclysmic variable or a sub-dwarf B star.

\section{References}

Bujarrabal, V., Alcolea, J., Van Winckel, H., Santander-García, M., \& Castro-Carrizo, A. 2013, $A \mathscr{E} A, 557, \mathrm{~A} 104$

Bujarrabal, V., Castro-Carrizo, A., Alcolea, J., \& Van Winckel, H. 2015, A\&̈A, 575, arXiv:1502.01607

de Ruyter S., Van Winckel H., Maas T., Lloyd Evans T., Waters L. B. F. M., Dejonghe H., 2006, A\& $A, 448,641$

Frogel J. A., Cohen J. G., Persson S. E., 1983, ApJ, 275, 773

Han Z., Podsiadlowski P., Eggleton P. P., 1995, MNRAS, 272

Hillen M. et al., 2013,A\&GA, 559, A111

Kamath, D., Wood, P. R., \& Van Winckel, H. 2014, MNRAS, 439, 2211

Kamath, D., Wood, P. R., \& Van Winckel, H. 2015, MNRAS, 454, 1468

Kamath, D., Wood, P. R., Van Winckel, H., \& Nie, J. D. 2016, A\&AA, 586, L5

Nie, J. D., Wood, P. R., \& Nicholls, C. P. 2012, MNRAS, 423, 2764

-. 2003, ARAA, 41, 391

Van Winckel, H., Lloyd Evans, T., Briquet, M., et al. 2009, A\&A, 505, 1221

Vassiliadis, E. \& Wood, P. R. 1993, ApJ, 413, 641

Nicholls, C. P., Wood, P. R., \& Cioni, M.-R. L. 2010, MNRAS, 405, 1770

\section{Discussion}

Q: Now sdB stars relate to your systems. Do you find similar period distribution?

De Marco: The final separation does not just depend on $\mathrm{q}=\mathrm{M}_{2} / \mathrm{M}_{1}$, but on the envelope binding energy. We reproduce quite a range of final separation in common envelope simulations. Another factor is how long, before the CE, the RLOF phase lasts and how much it depletes the envelope prior to the plunge. 